\title{
INTERNAÇÃO EM UMA UNIDADE DE EMERGÊNCIA HOSPITALAR: VIVÊNCIA
}

\author{
Lívia Moreira Barros', Thiago Moura de Araújo², Maria Fabiana de Sena Neri³ ${ }^{3}$ Enedina Soares4, \\ Joselany Áfio Caetano ${ }^{5}$
}

RESUMO: Objetivou-se descrever a vivência de familiares de pacientes atendidos em uma Unidade de Emergência de Fortaleza-Ceará acerca de sua colaboração nas atividades e dificuldades vivenciadas. Estudo descritivo, com abordagem qualitativa, foi desenvolvido com 34 familiares acompanhantes de pacientes que se encontravam em observação. Os dados foram coletados por meio de entrevistas com roteiro semiestruturado no período de outubro e novembro de 2010 e a análise foi feita de acordo com a temática de conteúdo. Os resultados denotam déficit de aprendizagem e de informações acerca do diagnóstico, tratamento e cuidados prestados ao paciente. As dificuldades vivenciadas referiram-se à comunicação, infraestrutura, privacidade e poluição sonora. Acredita-se que a equipe interdisciplinar e os dirigentes possam sanar as dificuldades por meio de ações administrativas, organizacionais e educativas.

DESCRITORES: Serviço hospitalar de emergência; Enfermagem em emergência; Cuidadores; Família.

\section{HOSPITALIZATION IN A HOSPITAL EMERGENCY UNIT: EXPERIENCES}

ABSTRACT: The aim was to describe the experience of family members of patients attended in an Emergency Unit in Fortaleza-Ceará regarding their collaboration in the activities, and the difficulties experienced. This descriptive study with a qualitative approach was undertaken with 34 family members accompanying patients under observation. The data was collected through interviews with semi-structured scripts in the period October - November 2010, and analysis was carried out in line with thematic content. The results denote shortcomings in learning and information regarding the diagnosis, treatment and care given to the patient. The difficulties experienced refer to communication, infrastructure, privacy, and noise pollution. It is believed that the interdisciplinary team and the directors may be able to remedy the difficulties through administrative, organizational and educational actions.

DESCRIPTORS: Hospital Emergency Service; Emergency nursing; Caregivers; Family.

\section{INTERNACIÓN EN UNA UNIDAD DE EMERGENCIA HOSPITALAR: VIVENCIA}

RESUMEN: La finalidad de este estúdio fue describir la vivencia de familiares de pacientes atendidos en una Unidad de Emergencia de Fortaleza, Ceará, acerca de su colaboración en las actividades y dificultades vividas. Es un estudio descriptivo, con abordaje cualitativo que fue desarrollado con 34 familiares acompañantes de pacientes que estaban en observación. Los datos fueron obtenidos por medio de entrevistas con guión semiestructurado en el periodo de octubre y noviembre de 2010 y el análisis, hecho de acuerdo con la temática de contenido. Los resultados apuntan déficit de aprendizaje y de informaciones acerca del diagnóstico, tratamiento y cuidados prestados al paciente. Las dificultades vividas se refieren a la comunicación, infraestructura, privacidad y polución sonora. Se cree que el equipo interdisciplinar y los dirigentes pueden sanar las dificultades por medio de acciones administrativas, organizacionales y educativas.

DESCRIPTORES: Servicio hospitalar de emergencia; Enfermería en emergencia; Cuidadores; Familia.

\footnotetext{
${ }^{1}$ Acadêmica de Enfermagem da Universidade Federal do Ceará - UFC. Bolsista CNPq de Iniciação Científica.

${ }^{2}$ Enfermeiro. Mestre em Enfermagem. Doutorando pelo Programa de Pós-Graduação em Enfermagem da UFC. Professor da Universidade Federal do Maranhão. ${ }^{3}$ Enfermeira. Especialista em Saúde da Família.

${ }^{4}$ Enfermeira. Doutora em Enfermagem. Livre-docente pela Universidade Estadual do Rio de Janeiro.

${ }^{5}$ Enfermeira. Doutora em Enfermagem. Professora do Programa de Pós-Graduação em Enfermagemda UFC.
} 


\section{INTRODUÇÃO}

A unidade de emergência, segundo a Organização Pan-Americana de Saúde, é destinada a promover serviços médicos requeridos com caráter de emergência e urgência para prolongar a vida ou prevenir consequências críticas, os quais devem ser proporcionados imediatamente. Diante dessa realidade, os usuários procuram os serviços hospitalares de urgência como uma das alternativas de acesso, pois entendem que eles reúnem um somatório de recursos, como consultas, remédios, procedimentos de enfermagem, exames laboratoriais e internações, que os tornam mais resolutivos ${ }^{(1)}$.

O encaminhamento para a unidade de emergência ocorre devido às situações que põem em risco a vida dos pacientes e, muitas vezes, o mantêm em estado grave, resultando em cobranças especiais quanto ao modo de cuidar realizado pela equipe de saúde. Diante da debilidade do doente, justifica-se a permanência de acompanhante, muitas vezes familiares, para auxiliar o paciente em suas necessidades ${ }^{(2-4)}$.

Um dos desafios no atendimento de urgência/ emergência é atender as expectativas dos pacientes e familiares, pois quando um familiar recebe a notícia de que seu parente necessita ser internado na unidade de emergência, ocorre uma sensação de estranheza e impotência, permeada de medos e ansiedades, culminando com o desequilíbrio emocional, desestabilização e crise familiar ${ }^{(1)}$. E, muitas vezes, o processo de trabalho da equipe de saúde tem como foco as ações procedimentais, deixando o desejo e o aspecto humano do paciente, e de sua família, num plano secundário de atenção.

A enfermagem, independentemente do local de atendimento ao cliente, precisa valorizar e reconhecer a importância da família. Pois, todo indivíduo faz parte de uma conjuntura que foi modificada por algum motivo externo e que pode determinar repercussões não favoráveis à saúde individual, familiar e coletiva ${ }^{(5)}$. A permanência de familiares acompanhantes junto ao doente hospitalizado tem exigido transformações na prática da equipe de enfermagem. Deste modo, a equipe necessita adaptar-se a esta situação, alterando atitudes e posturas, demonstrando receptividade frente à presença do acompanhante no cotidiano do cuidado ${ }^{(6)}$. O acompanhante no cenário estressante e conflituoso desse setor necessita de apoio e solidariedade da equipe multiprofissional para o enfrentamento do momento ${ }^{(4)}$.

Dessa forma, diante da permanência da família ao lado do paciente e de sua participação no cuidado, da importância da identificação, pelos enfermeiros, dos cuidados realizados e conhecimento das reais necessidades dos acompanhantes com vistas a desenvolver ações para a promoção da saúde dos pacientes, este estudo foi realizado com o objetivo de descrever a vivência de familiares de pacientes atendidos em uma unidade de emergência a respeito da colaboração destes nas atividades prestadas e das dificuldades vivenciadas.

\section{MÉTODO}

Estudo descritivo, com abordagem qualitativa, realizado de outubro a novembro de 2010 , em um hospital de alta complexidade, de nível quaternário, em Fortaleza-Ceará. O estudo descritivo busca descrever características de uma população ou fenômeno ${ }^{(7)}$. Optou-se por este tipo de pesquisa por considerar que o objeto em estudo busca, através da subjetividade dos familiares acompanhantes, apreender a vivência dos familiares a respeito de sua colaboração nas atividades prestadas nesses serviços e as dificuldades vivenciadas.

Os participantes do estudo foram 34 familiares acompanhantes, os quais atenderam aos critérios de inclusão propostos: ser maior de 18 anos e estar acompanhando o doente atendido e em observação na emergência por um período entre 24 e 48 horas. Justificamos este período por concordância com as recomendações de que nos casos de urgência ou emergência não se admitiria que o paciente permanecesse na unidade por mais de 24 horas, ou em casos especiais, mais de 48 horas $^{(8-9)}$. Para a coleta de dados foi utilizado um roteiro previamente elaborado contendo quatro questões norteadoras: Quais as atividades que você, na qualidade acompanhante, tem colaborado durante a hospitalização de seu familiar? Quais as dificuldades que você tem ao realizar essas atividades? O que você sente ao colaborar nas atividades junto ao seu familiar? O que gostaria de saber em relação ao seu acompanhamento como familiar?

Para análise das narrativas procedeu-se à transcrição na íntegra das entrevistas, leitura e releitura do conteúdo de cada entrevista e agrupamento em quatro categorias de análise procurando identificar as unidades de significado definidoras, denominadas neste estudo como 'classes', nessa análise temática de conteúdo. A técnica de análise seguida ocorreu conforme as fases de análise, cujos passos foram: ordenação dos dados, classificação dos dados e discussão final ${ }^{(10)}$.

O projeto foi aprovado pela pelo Comitê de Ética em Pesquisa da instituição em que foi realizada a pes- 
quisa sob protocolo n. 110904/08. Após esclarecimento sobre os objetivos e finalidades da pesquisa, todos os convidados aceitaram participar do estudo e assinaram o Termo de Consentimento Livre e Informado. Para manter o anonimato dos participantes, os registros foram identificados pela letra "F" (familiar), seguida do número que correspondeu à ordem da entrevista (de 1 a 34).

\section{RESULTADOS}

Foram entrevistados 34 familiares acompanhantes. Houve prevalência do sexo feminino, 27 eram mulheres e sete homens, na faixa etária entre 18 e 35 anos. Com relação à escolaridade, 23 familiares possuíam o ensino médio completo, cinco não eram alfabetizadas, três concluíram o ensino fundamental, dois familiares tinham o ensino profissionalizante e apenas um concluiu o ensino superior. Quanto ao estado civil, 21 eram casados, 11 solteiros, um viúvo e um divorciado. No tocante à ocupação, 13 eram domésticas, três agricultores, seis aposentados e 12 não tinham profissão definida. Quanto à renda, 17 recebiam um salário mínimo regional, seis ganhavam menos de um salário mínimo e 11 não tinham renda. Com relação ao vínculo familiar, 18 eram filhos(as), 13 esposos(as) e três irmãs.

Os depoimentos sobre a vivência de familiares de pacientes a respeito da colaboração destes nas atividades prestadas, e as dificuldades, estão apresentados, por meio de recortes das falas, com a relativa categoria, no quadro 1.

Quadro 1 - Correlação entre categorias e as falas dos familiares cuidadores em uma unidade de atendimento de emergência. Fortaleza, 2010

\begin{tabular}{|c|c|}
\hline CATEGORIAS & FALAS DOS PARTICIPANTES \\
\hline $\begin{array}{l}\text { Dificuldades dos familiares } \\
\text { cuidadores em estar e ajudar } \\
\text { na emergência }\end{array}$ & $\begin{array}{l}\text { Falta de privacidade, barulho excessivo, falta de informação com relação } \\
\text { aos exames. Banheiro sem rampa, com água empossada e não tem como } \\
\text { pendurar toalha e soro. (F 9) }\end{array}$ \\
\hline $\begin{array}{l}\text { Acompanhantes cuidadores e } \\
\text { seus sentimentos }\end{array}$ & $\begin{array}{l}\text { [choro e silêncio] eu não queria que a minha forma de gratidão fosse re- } \\
\text { tribuida assim, ter que cuidar dele doente. (F 19) } \\
\text { Prazer, alegria, paz, uma grande vitória [...]. Hoje ela está falando. } \\
\text { Sinto-me muito bem; faço porque gosto, prá mim eu estou salvando, } \\
\text { valorizando a vida. (F25) } \\
\text { Faço tudo com amor e carinho. Sinto, às vezes, medo, pena e tristeza. Faço } \\
\text { parte da recuperação dele, sou tocada a cuidar, adio meus compromissos, } \\
\text { quando posso. (F 17) }\end{array}$ \\
\hline $\begin{array}{l}\text { Necessidade de informação do } \\
\text { cuidador }\end{array}$ & $\begin{array}{l}\text { Quero saber por que não pode beber, quais as reações que fazem parte do } \\
\text { problema e quais são os sinais de perigo. (F18) } \\
\text { Informações sobre higiene pessoal e do ambiente, fumo, lavagem das mãos } \\
\text { para acompanhante. (F23) } \\
\text { Quais os exercícios que posso fazer com ele e informação sobre curativo. } \\
\text { (F21) } \\
\text { O que precisa ou não comer, saber sobre o seu cansaço. (F 26) } \\
\text { Queria que dissesse qual é o problema, se é ruim ou o que é! Como é o } \\
\text { tratamento, como ele poderia ficar bom. (F 24) } \\
\text { Gostaria de saber se ele vai se operar, se ele tem cura, quais os remédios } \\
\text { que ele toma, não sei para que servem os exames que ele fez. (F 19) } \\
\text { Eu não sei quais as reações da hemodiálise, informações quanto ao que } \\
\text { vão fazer com ele e o que esperar. (F13) } \\
\text { Será que vai deixar sequelas? Devido a doenças vai precisar de fisiotera- } \\
\text { pia? Se a doença dela pode se agravar. (F19) } \\
\text { Não sei se vai ser preciso tirar o dedo, se o doutor vem hoje. Deixa a } \\
\text { desejar a falta de informação para cuidar melhor. Não informam quais } \\
\text { os materiais para curativo e o que pode ou não fazer no hospital (F 14) }\end{array}$ \\
\hline
\end{tabular}

Cogitare Enferm. 2013 Abr/Jun; 18(2):336-43 
Das dificuldades citadas acerca das atividades executadas pelos familiares, independentemente ou sob supervisão da enfermagem, as mais apontadas foram: comunicação entre os profissionais de saúde, ausência de privacidade, desinformação, barulho, número insuficiente de profissionais para atender à demanda $\mathrm{e}$ estrutura física deficiente.

Quando questionados sobre o que sentiam ao desempenhar as atividades junto ao familiar hospitalizado, os sentimentos foram expressos sob a forma de gratidão, de dever, de recompensa pela possível melhora no estado de saúde da pessoa adoentada, de estar ajudando, de tristeza, de alívio e de importância para pessoa da qual cuidava.

Quanto ao que gostariam de saber com relação às funções que poderiam colaborar como familiares cuidadores junto aos clientes em processo de atendimento na emergência, observou-se, primeiramente, que apresentavam dificuldades e que gostariam de adquirir conhecimentos para atender melhor ao familiar enfermo. Os familiares cuidadores também solicitaram informações sobre a doença, desde o diagnóstico médico até o prognóstico e acerca de procedimentos que poderiam ser realizados por eles.

\section{DISCUSSÃO}

$\mathrm{Na}$ sociedade, quem assume o cuidado do doente é a família, a qual tem papel fundamental no cuidar, visto que ela constitui uma referencia para o individuo enfermo $^{(7)}$. Estudos têm demonstrado que, entre os membros familiares, os que mais têm ocupado o papel de cuidador são mulheres, notadamente filhas, netas, sobrinhas, esposas e irmãs. Quando um familiar adoece, dificilmente um homem assume os cuidados diretos, ele pode contribuir secundariamente, com transporte, promoção de atividades sociais, questões legais, dentre outras. Porém, a experiência de cuidar geralmente está associada à figura da mulher na família, cuidando de outras pessoas, doentes ou não, crianças ou adulto ${ }^{(8)}$. $\mathrm{O}$ predomínio do sexo feminino é compatível com outros estudos realizados com acompanhantes. Estudo realizado com cuidadores de pacientes portadores de Acidente Vascular Cerebral identificou 67,5\% do sexo feminino, $48 \%$ filhas dos pacientes e $38,3 \%$ estando entre 31 e 44 anos $^{(9)}$. Outra pesquisa realizada com acompanhantes de pacientes hospitalizados em clínica médica e cirúrgica verificou 8 mulheres num total de 9 participantes.

A faixa etária dos acompanhantes cuidadores esteve situada no grupo de pessoas socialmente ativas. $\mathrm{O}$ acompanhamento desses pacientes pode ser identificado como problema, pois os parentes tem que se afastar de suas rotinas de trabalho e afazeres domésticos para atender às necessidades do paciente em tempo integral. A responsabilidade desses acompanhantes também é percebida quando se analisa o tipo de união. A maioria encontrava-se casada, o que compromete as demandas no lar e, consequentemente, pode haver cobrança pela (o) parceira (o), não compreendendo a ausência do outro(11).

Neste estudo, a maioria dos cuidadores tinha baixa escolaridade e apenas um familiar concluiu o ensino superior, o que divergiu dos resultados encontrados em outro estudo realizado em um hospital da Região Sul do Brasil verificou-se que 46,6\% dos acompanhantes possuíam ensino superior completo. Salienta-se que a maior qualificação favorece a prestação dos cuidados, devido ao acesso às informações, apesar de ter sido observado que o estresse de cuidar atinge a todos os acompanhantes e cuidadores, indistintamente ${ }^{(8)}$.

Entre os participantes, a renda familiar mensal máxima foi de um salário mínimo. A ausência de atividade remunerada, devido à responsabilidade com o familiar enfermo, acentua a situação de precariedade econômica da família, o que pode contribuir para a inadequação do cuidado ${ }^{(8)}$.

\section{Dificuldades dos familiares/acompanhantes em estar e ajudar na emergência}

A unidade de atendimento de emergência é permeada de condições complexas, inerentes ao próprio ambiente e aos indivíduos presentes, os quais cuidam e são cuidados e vivenciam as complexas relações humanas no processo de cuidar ${ }^{(9)}$. Por isso, no ambiente emergencial são encontrados problemas básicos como demora no atendimento e carência de profissionais, os quais põem em risco a qualidade do atendimento e, consequentemente, o processo de humanização da assistência ${ }^{(4)}$.

$\mathrm{O}$ familiar que acompanha o paciente em unidades de emergência precisa se adaptar a um cenário geralmente hostil, que não the promove conforto. Quando se depara com circunstâncias decorrentes de uma nova situação, como ausência de leito para repouso, precisando se adaptar à rotina hospitalar, conviver com outras experiências de sofrimento e tendo que se acostumar às situações imprevisíveis que, ainda, assumindo um papel de ter que colaborar nos cuidados.

No momento da hospitalização e do processo de permanência no ambiente hospitalar, a enfermagem, ao realizar o acolhimento ao familiar, permite que o encontro se estabeleça e o diálogo ocorra. O estar presente, o relacionar-se, a criação de vínculo entre 
familiar/enfermagem são formas de se estabelecer as relações de acolhimento ${ }^{(12)}$. A equipe de enfermagem deve integrar os acompanhantes ao tratamento do seu familiar, explicando cada procedimento a ser efetuado e mostrando como ele pode ajudar, pois é com a sua participação que se determina melhor aceitação ao tratamento e recuperação do cliente ${ }^{(5)}$.

Independente do estado de saúde do cliente ser crítico, ou não, as ações e relações de cuidado na unidade de emergência ocorrem naturalmente, num processo de mútua troca entre o profissional de enfermagem e o cliente, considerando-se as singularidades e também pluralidade dos seres, as experiências vividas e adquiridas, isolada ou coletivamente ${ }^{(9)}$.

Para que as intervenções de enfermagem em uma unidade de emergência sejam eficientes e eficazes, $o$ enfermeiro precisa valorizar também a subjetividade do ser humano. É de competência da enfermagem o cuidado ao paciente, o conforto, o aconchego, a calma e a tranquilidade, bem como adequadas condições de higiene e limpeza do ambiente ${ }^{(9)}$. Nesse contexto, insere-se, também, o familiar como colaborador no cuidado, pois o mesmo também necessita de cuidado, pelo menos em nossa concepção.

\section{Acompanhantes cuidadores e seus sentimentos}

A hospitalização é um desafio não só para o paciente, como também para a família. Esta se sente aflita com a situação do seu parente, que se encontra em estado crítico. As dúvidas sobre sua doença e seu prognóstico geram na família um sentimento de preocupação ${ }^{(13)}$. $\mathrm{O}$ acompanhante, quando fragilizado pelo medo de uma possível perda, e pela aflição de ver o familiar em situação crítica, recorre à equipe de enfermagem para compartilhar seus sentimentos e preocupações ${ }^{(5)}$.

Por meio das verbalizações dos familiares, o cuidado exercido por eles torna-se prazeroso, devido aos laços afetivos existentes, que se fortalecem na oportunidade de retribuir. É uma forma de gratidão, amor, carinho. Ainda, motivam e explicam a atitude de assumir o cuidar, inclusive adiando outros afazeres.

Muitos familiares cuidadores percebem o cuidado como algo que os dignifica como pessoa ao cumprir um dever moral e religioso, o qual traz satisfação pela manifestação de gratidão do familiar doente e pelo reconhecimento da família e da comunidade ${ }^{(14)}$.

O familiar vivencia a sobrecarga física, emocional e socioeconômica, sendo fundamental que ele se torne mais seguro e preparado para assumir as responsabili- dades no cuidado do familiar dependente. A família, além de passar pelas mudanças causadas pelo processo de adoecer do seu parente, ainda se depara com um ambiente até então estranho, com pessoas desconhecidas, onde se executa procedimentos diversificados e, muitas vezes, incompreensíveis, os quais geram expectativas e sentimentos de ansiedade ${ }^{(13)}$.

Os familiares demonstram preocupação quanto à doença e prevenção, ao associarem os vícios com a possível doença de seu familiar. Isso é positivo à medida que tenham orientações para evitar riscos e ou complicações. Logo, a emergência se configura como local ideal de orientações para ações de prevenção ou que evite agravamento da doença. Identificam-se orientações voltadas para o controle de infecção hospitalar, com medidas de precaução de contato ${ }^{(15-17)}$.

\section{Necessidade de informação do cuidador}

Essa questão mostrou que a população pesquisada estava aberta para receber informações, objetivando conhecer detalhadamente o quadro clínico do familiar. Em suma, as necessidades encontradas de aquisição de orientações por parte dos cuidadores relacionavam-se às doenças e ao tratamento. A saúde tem procurado avanços para atender à população em geral, com novas tecnologias e habilidades para diminuir o sofrimento do paciente, diminuir o tempo de tratamento e aumentar suas possibilidades de cura. Por se tratar de um momento de difícil enfrentamento, é importante que as dúvidas dos familiares sejam esclarecidas, a respeito do estado do paciente e do tratamento que ele está recebendo, para que, dessa forma, sentimentos como a preocupação sejam minimizados ou, até mesmo, eliminados $^{(13)}$.

Nas falas dos familiares, evidenciou-se o aspecto da tentativa, no sentido de não delegar atribuições exclusivas da enfermagem a terceiros, com risco para a equipe e o paciente. Outra preocupação que deve ser enfocada é em razão de familiares, com intenção de ajudar, mas desprovidos de conhecimento científico, de realizar procedimentos que possam pôr em perigo o paciente e seu tratamento ${ }^{(14)}$.

A informação oferecida ao familiar é muito importante, sobretudo para conhecer o motivo da internação, o que se faz para os clientes internados e como é a dinâmica do trabalho dos funcionários da unidade. $\mathrm{O}$ familiar precisa estar seguro de que a pessoa internada receberá toda a assistência de que necessita ${ }^{(5)}$. A pessoa bem informada facilita e auxilia no tratamento 
de saúde. Informar ao familiar e ao cliente sobre o processo saúde/doença é de suma contribuição para o bom trabalho dos profissionais e da instituição, principalmente na emergência, em que a informação e a consequente ação podem salvar vidas humanas. Além disso, as informações, dadas de forma esclarecedora, podem ser interpretadas pelos familiares como resposta do enfermeiro às preocupações, dúvidas e medos ${ }^{(18)}$.

Uma das dificuldades dos familiares cuidadores é de entender a doença e suas complicações, ficando em dúvida se está prestando o cuidado de forma adequada ${ }^{(14)}$. Está em poder da enfermagem, enquanto profissão que enfatiza o tratamento personalizado e holístico, realizar as ações que podem viabilizar o entendimento e a compreensão da internação, tanto por parte dos sujeitos nela internados quanto de seus familiares ${ }^{(12)}$.

Neste estudo, a maior preocupação manifestada pelas cuidadoras, em relação ao cuidado, foi sobre o problema de saúde, se tinha cura, causa, o que fazer e o risco de agravamento do quadro. Essas preocupações podem ser atribuídas à insegurança e aos insuficientes conhecimentos básicos de saúde, por parte dos familiares cuidadores. Assim, essas características se correlacionam com a falta de suporte e de educação em saúde para os cuidadores.

Algumas das atividades em que o familiar cuidador dedica a atenção ao paciente são auxílio na deambulação e deslocamento, auxílio durante o banho, encaminhamento ao sanitário, auxílio em situações de incontinência urinária, ajuda no vestir-se/despir-se, alimentar-se, entre outras ${ }^{(15)}$. Dessa forma, o desejo de aprender referiu-se a cuidados de atividades da vida diária, como: alimentação, ingestão de líquidos, higiene, exercícios, os quais são diferentes quando a pessoa está doente num ambiente hospitalar. Por exemplo, ingestão de líquidos ou a dieta zero para o cliente que se encontra em esclarecimento de diagnóstico ou aguardando cirurgia. Esses cuidados são observados e têm implicações com prejuízo ao tratamento. Então, os cuidadores têm razão em perguntar quando percebem que esses ensinamentos não são repassados para eles, como acompanhante do paciente. A comunicação entre os acompanhantes e profissionais de saúde torna-se de extrema importância para detectar os anseios e as apreensões do paciente, refletidos pela fala dos cuidadores ${ }^{(11,14)}$.

Entre o diagnóstico e o tratamento permeia a realização de exames, uso de curativos ou sondas e administração de remédios. Foram esses procedimentos e suas finalidades que os familiares demonstraram estar desejosos em aprender e expressaram isso enfaticamente nos discursos. Portanto, torna-se importante conhecer o diagnóstico do paciente, bem como o prognóstico em relação ao processo de saúde/doença. A realização de exames diagnósticos está incluída na sequência do atendimento realizado pela equipe multiprofissional. Estudo realizado em Santa Catarina, com o objetivo de compreender o significado das relações de cuidado de enfermagem em uma unidade de emergência na percepção do ser humano cuidado, observou que os pacientes consideram importante realizar exames diagnósticos, pois os mesmos se sentem cuidados e valorizados e consideram que essa ação possibilita e favorece a intervenção terapêutica ${ }^{(9)}$.

O profissional de enfermagem, ao atuar em unidade crítica de saúde, deve demonstrar destreza, agilidade, habilidade, bem como, capacidade para estabelecer prioridades e intervir de forma consciente e segura no atendimento ao ser humano, sem esquecer que, mesmo na condição de emergência o cuidado é o elo de interação/integração/relação entre profissional e cliente ${ }^{(9)}$. A enfermagem deve investir cada vez mais no processo de comunicação, uma vez que este subsidiará o cuidado efetivo e eficaz ao cliente hospitalizado. A busca e o resgate de sentimentos, valores e atitudes entre equipe de enfermagem e familiares são os caminhos nos quais se pode alcançar satisfação pela assistência prestada durante o período de tratamento na instituição de saúde ${ }^{(5)}$. Reconhece-se, assim, a necessidade de intervenção dos profissionais através da comunicação.

Quando a hospitalização se faz necessária, o indivíduo é retirado de seu ambiente familiar, no hospital encontra rotinas e normas rígidas que controlam e determinam suas ações, em que comunicação não efetiva poderá retardar o tratamento. Mesmo neste ambiente inóspito, em todas as situações de contato está presente a comunicação cliente/enfermagem/ acompanhante $^{(19)}$. A dificuldade de relacionamento entre a enfermagem e a família induz ao distanciamento. Esse ocorre por vários fatores, os mais evidentes referem-se à falta de preparo da enfermagem frente às necessidades da família e à organização do traba1ho. Estabelecer vínculo enfermagem/família é uma forma de amenizar o isolamento social, bem como auxiliar na reestruturação biopsicossocial da família ${ }^{(12)}$.

O cuidado empírico do familiar cuidador não vem avançando na mesma proporção. As necessidades desses familiares cuidadores são reflexos de um atendimento voltado para o modelo biomédico, sem atender às necessidades do pacientes como um todo. Devido a esta realidade, atuar de forma humana na sala de emergência é um desafio ao enfermeiro e sua equipe ${ }^{(20)}$. 


\section{CONSIDERAÇÕES FINAIS}

Propor-se deste estudo identificar os fatores que afetam os familiares acompanhantes de pacientes atendidos em uma unidade de emergência e sua participação nas atividades prestadas nesses serviços e das dificuldades vivenciadas.

As dificuldades que o familiar apresentou ao realizar as atividades de colaborador no cuidado foram relativas a comunicação, infraestrutura, pouca privacidade e poluição sonora e insuficiência de informações. Entretanto, os familiares relataram a necessidade de recompensa, expressa pela melhora no estado de saúde do familiar que estava dependente de cuidados. A discussão sobre a importância da relação entre paciente/acompanhante e o profissional deverá permear as demais profissões, promovendo visão multiprofissional sobre essa problemática. As dificuldades reconhecidas neste estudo pelos acompanhantes podem refletir aspectos estruturais e políticos. Acreditamos, portanto, que a equipe interdisciplinar e os dirigentes institucionais possam tentar sanar essas dificuldades, com a contribuição a partir da reforma do ambiente hospitalar e mudanças de comportamento perante o cliente e seus familiares.

\section{REFERÊNCIAS}

1. Marques GQ, Lima MADS. Demandas de usuários a um serviço de pronto atendimento e seu acolhimento ao sistema de saúde. Rev. Latino-Am. Enfermagem. 2007;15(1):13-9.

2. Barbosa KP, Silva LMS, Fernandes MC, Torres RAM, Souza RS. Processo de trabalho em setor de emergência do hospital de grande porte: a visão dos trabalhadores de enfermagem. Rev Rene. 2009;10(4):70-6.

3. Sarti CA. O atendimento de emergência a corpos feridos por atos violentos. Physis. 2005;15(1):107-26.

4. Andrade LM, Costa MFM, Caetano JA, Beserra EP. A problemática do cuidador familiar do portador de acidente vascular cerebral. Rev Esc Enferm USP. 2009;43(1):37-43.

5. Schimidt TCG, Arruda ML. Sentimentos da família na interação com a equipe de enfermagem. Cogitare enferm. 2012;17(2):348-54.

6. SzareskiC,BeuterM,BrondaniCM.Ofamiliaracompanhante no cuidado ao adulto hospitalizado na visão da equipe de enfermagem. Rev. Gaúcha Enferm. 2010;31(4):715-2.
7. Oliveira SG, Quintana AM, Budo MLD, Ludtke MF, Cassel PA, Wottrich SH. Familiares cuidadores e a terminalidade: tendência da produção cientifica na área de saúde. REME: rev. min. enferm. 2011;15(4):588-94.

8. Arruda MC, Alvarez AM, Gonçalves LHT. O familiar cuidador de portador de doença de Alzheimer participante de um grupo de ajuda mútua. Cienc. cuid. saude. 2008;7(3): 339-45.

9. Baggio MA, Callegaro GD, Erdmann AL. Compreendendo as dimensões de cuidado em uma unidade de emergência hospitalar. Rev Bras Enferm. 2009;62(3):381-6.

10. Polit DF, Beck CT. Fundamentos de pesquisa em enfermagem: métodos, avaliação e utilização. $7^{\mathrm{a}} \mathrm{ed}$. Porto Alegre: Artmed; 2011.

11. Vieira GB, Alvarez AM, Gonçalves LTI. A enfermagem diante dos estressores de familiares acompanhantes de idosos dependentes no processo de hospitalização e alta. Cienc. cuid. saude. 2009;8(4):645-51.

12. Frizon G, Nascimento ERP, Bertoncello KCG, Martins JJ. Familiares na sala de espera de uma unidade de terapia intensiva: sentimentos revelados. Rev. Gaúcha Enferm. 2011;32(1):72-8.

13. Almeida AS, Aragão NRO, Moura E, Lima GC, Hora EC, Silva LASM. Sentimentos dos familiares em relação ao paciente internado na unidade de terapia intensiva. Rev Bras Enferm. 2009;62(6):844-9.

14. Escher RB, Cogo ALP. Os familiares de pacientes adultos hospitalizados: sua participação no processo de cuidar na enfermagem. Rev. Gaúcha Enferm. 2005;26(2):242-51.

15. Pimenta GMF, Costa MASMC, Gonçalves LHT, Alvarez AM. Perfil do familiar cuidador de idoso fragilizado em convívio doméstico da grande região do Porto, Portugal. Rev Esc Enferm USP. 2009;43(3):609-14.

16. Bocchi SCM, Silva L, Juliani CMCM, Spiri WC. Familiares visitantes e acompanhantes de adultos e idosos hospitalizados: análise da experiência na perspectiva do processo de trabalho em enfermagem. Rev. Latino-Am. Enfermagem. 2007;15(2):304-10.

17. Pena SB, Diogo MJDE. Fatores que favorecem a participação do acompanhante no cuidado do idoso hospitalizado. Rev. Latino-Am. Enfermagem. 2005;13(5):663-9. 
18. Maestri E, Nascimento ERP, Bertoncello KCG, Martins JJ. Estratégias para o acolhimento dos familiares dos pacientes na unidade de terapia intensiva. Rev. enferm. UERJ. 2012;20(1):73-8.

19. Squassante ND. A dialética das relações entre a equipe de enfermagem e familiares acompanhantes no hospital: implicações para o cuidado de enfermagem [dissertação]. Rio de Janeiro (RJ): Universidade Federal do Rio de Janeiro; 2007.

20. Montezeli J, Meier M, Peres A, Venturi K, Wolff L. Enfermagem em emergência: humanização do atendimento inicial ao politraumatizado à luz da teoria de Imogene King. Cogitare enferm. 2009;14(2):384-7. 